\title{
Office of Cancer Survivorship
}

National Cancer Institute

\section{Source}

National Cancer Institute. Office of Cancer Survivorship. NCI Thesaurus. Code C19774.

The Office of Cancer Survivorship was established in 1996 to provide support and a focus for research and other activities dealing with cancer survivors. OCS workshops were held in 1996 and 1997 to define priorities for research, which included the prevalence of physical effects from cancer treatment, the prevalence of second cancers in survivors, quality of life, and quality and cost of follow-up care for survivors. 